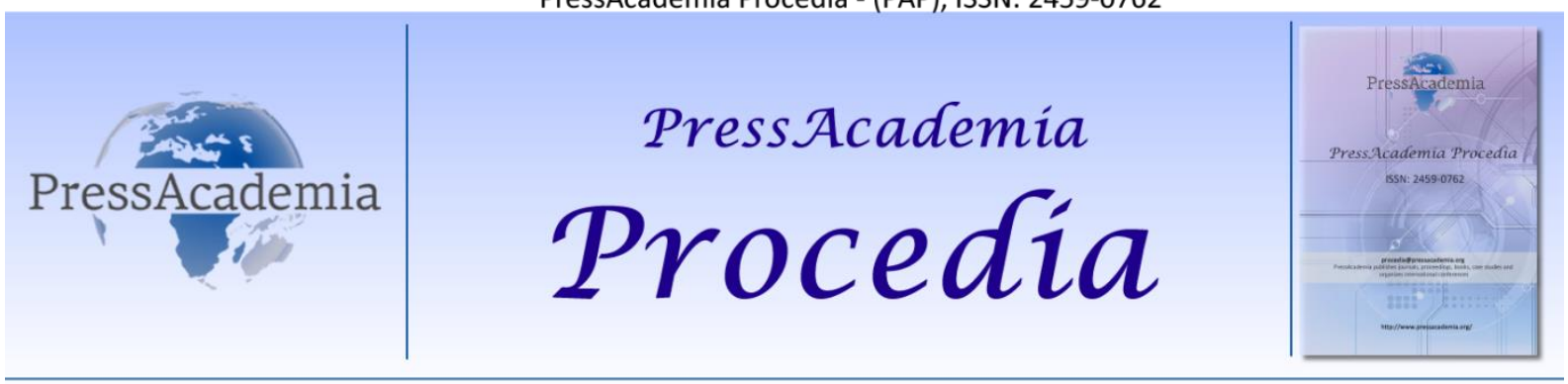

2nd World Conference on Technology, Innovation and Entrepreneurship

May 12-14, 2017, Istanbul, Turkey. Edited by Sefer Şener

\title{
HOMEWORK SYSTEM DEVELOPMENT WITH THE INTENTION OF SUPPORTING SAUDI ARABIA'S VISION 2030
}

\author{
DOI: 10.17261/Pressacademia.2017.616 \\ PAP-WCTIE-2017(54)-V.4-p.397-401
}

Atifa Elgimari ${ }^{1}$, Shafya Alshahrani ${ }^{1}$ and Amal Al-shehri ${ }^{1}$

${ }^{1}$ Department of Computer Science, University of Bisha, Bisha- Kingdom of Saudi Arabia, atfa000@gmail.com

\begin{abstract}
This paper suggests a homework system based developing a website. By using the suggested homework system, hard copies of homeworks were replaced by soft copies. It is expected to participate in applying Saudi Arabia's Vision 2030, specially in the education sector, where it considers that the primary education is its foundation stone, as the success of the Vision depends in large assess on reforms in the education system generating a better basis for employment of young Saudis.
\end{abstract}

Keywords: Homework, teacher, parent, education, system, vision

\section{INTRODUCTION}

Homework is a long-standing education tradition that, until recently, has hardly ever been questioned. It has many types of definitions; it is defined as out-of-class tasks assigned to students by their teachers as amplification or an extension of classroom. Homework is a headache for most parents because it is a daily obligation [3]. The author [2] discussed how to advice parents to be powerful leaders in their kid's education. He answered the most common questions parents ask such as When do I reward and when do I punish?, How do I give advice about studying without getting backtalk?, How much should I review homework?, How do I help my stressed out child who takes school too seriously?

What do I say when my child says "I don't care" about school?, How do I help my disorganized child without feeling like I am his secretary?, and so on.

A popular book [1] introduced the term homework from the respect of recent changing in culture and in the different societies. However, Internet and bookstores are full of books offering parents advice on how to get children to do homework, but still homework has become problematic for more and more students, parents, and teachers. As the culture has changed, and as schools and families have also changed.

For sure homework places great importance in the educational process, moreover, [5] considered homework as a base for the learning process. The Ministry of Education in Saudi Arabia, general education sector, annually provides students with hard copies of homeworks note for free. Nevertheless that costs the Ministry of Education a huge amount of money. This paper suggests a homework system based on developing a website application. By using the suggested homework system, hard copies of homeworks are replaced by soft copies. From different viewpoint, parents were not involved in the educational process over the past few years. Recently, involving parents in children's education is being placed in the educational process. The suggested homework system engages parents and teachers on a day-to-day basis. Teachers can send homework to the system; parents can browse them, therefore they encourage their children to do their homework.

The objective of this paper is to provide a framework for a homework system that would help teachers and parents in collaborating goals that best fit students' achievement. The suggested homework system is expected to participate in 
applying Saudi Arabia's Vision 2030, which it considers a cornerstone of education. The success of the Vision depends on large assess on reforms in the education system generating a better achievement.

\section{SAUDI ARABIA's VISION 2030}

Vision 2030 was approved by the Saudi cabinet on 25-April-2016, which confines the objectives of the government for the coming 15 years. According to reference [4], the vision carefully addresses three major themes (society, economy, nation), and assigns goals to each sub-theme, then concludes with commitments for each theme. Among the commitments that are applicable to education, research, and renewable energy. The vision gives young Saudi students extraordinary significance. It plans to build a centralized student database tracking students from early childhood through to K-12 and beyond into tertiary education (higher and vocational).

Vision 2030's view about the transformation process of Education in Saudi Arabia, its goal is to draw their attention to attract and retain the finest Saudi and foreign minds, and facilitate all needed requirements for them, in addition, to provide them with all they need.

To this end, and for getting excellent impacts, we will prepare a modern curriculum focused on rigorous standards in literacy, numeracy, skills and character development. The vision will track progress and publish a sophisticated range of education outcomes, showing year-on-year improvements. It will work closely with the private sector to ensure higher education outcomes are in line with the requirements of the job market [6]. Additionally, The vision will continue to improve and reform their regulations, paving the way for investors and the private sector to acquire and deliver services such as...education - that are currently provided by the public sector. The vision will look to shift the government's role from providing services to focusing on regulating and monitoring the educational system. It will build the capability to monitor this transition.

Saudi Arabia's Vision 2030 sets out a motivated road-map for education development in the Kingdom of Saudi Arabia. This road-map reflects a collective vision for developing curricula, teaching methods, students' values and institution skills.

\section{COLLECTING DATA}

No doubt good data collection is the foundation, on which good research is built [7].

Our group was enabled to gather data information through interviews of respondents from the different schools. The respondents included teachers, psychologists, and socialists. This was used as the primary method in collecting data on the preferred use of the end-user on the supposed system.

All of this information was gathered in order to be of help in comparing between the current system and the suggested homework system.

We had a discussion and shared different ideas in sequence to decide the best way to develop the suggested homework system. The choices were, developing a website or developing a mobile application. After discussion and review of the data, the decision was to build a website. We also decided the programming language to be used and other applications and materials needed in turn to make the suggested system possible.

In addition to interviews, the data on which this study was based was obtained from a questionnaire. The questionnaire was distributed over different types of social media sites.

\section{RESULTS}

The questionnaire conducted in a nationwide of a randomly selected sample of 377 persons. $\quad 41.8 \%$ out of them were teachers, and $58.2 \%$ were parents. Regardless of Poll, (Fig.1) the majority members of the random selected sample were parents. Respondents completed an extensive questionnaire whose main issue of investigation was a comparison between the current system and our suggested system. In respect of using the current system, we examined the effect of handwriting notes of homeworks on lecture time. $85 \%$ of teachers answered that handwriting notes are time consuming (Fig.2). Specifically, we related these answers to the benefits of using the suggested system; it was found that $87.4 \%$ out of 127 teachers' responses presented their willingness to use the suggested homework system (Fig.3). Comparing with $92 \%$ out of 177 parents' responses presented their willingness to use the suggested homework system (Fig.5).

The data assembled asked parents many questions such as "has it happened that your son/daughter has forgotten his/her homework note, and that reflected negatively on his/her academic achievement?" Poll shows that about 39\% of parents have agreed, $28.8 \%$ disagreed, $32.2 \%$ said sometimes out of 177 respondents (Fig.4). 
The suggested homework system will empower parents and teachers in collaborating together in order to improve students' achievement.

Figure 1:" Is the responder a teacher or a parent?"

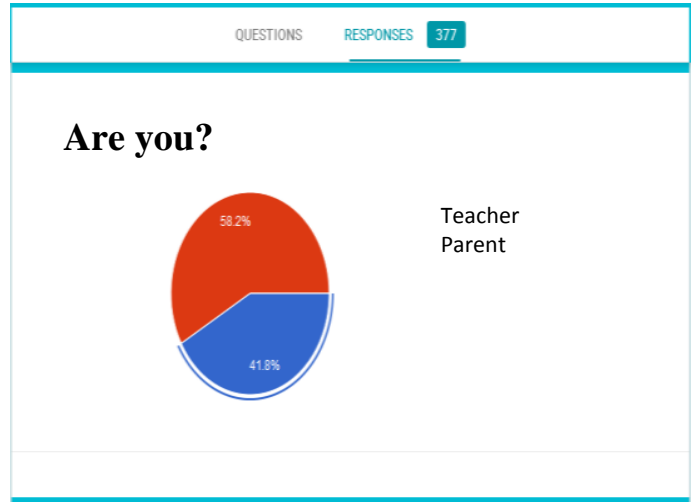

Figure 2: Questionnaire asked teacher" Do you think that handwriting notes of homework is time consuming for lecture time?"

\begin{tabular}{|l|}
\hline Dear Teacher \\
\hline $\begin{array}{l}\text { Do you think that handwriting notes of } \\
\text { homework is time consuming for lecture time? }\end{array}$ \\
\hline $\begin{array}{l}\text { No } \\
\text { Nometimes }\end{array}$ \\
\hline
\end{tabular}

Figure 3: Questionnaire asked teacher "Are you going to use our suggested system?"

\begin{tabular}{|l|l|}
\hline \multicolumn{1}{|c|}{ Dear Teacher } \\
\multicolumn{2}{|c|}{$\begin{array}{l}\text { Questions } \\
\text { Are you going to use our suggested } \\
\text { system? }\end{array}$} \\
\hline
\end{tabular}


Figure 4: Questionnaire asked parent "Had it happened that your son/daughter forgotten his/her homework note, and that reflected negatively on his/her academic achievement?"

\section{Dear Parent}

Has it happened that your son/daughter forgot his/her homework note, and that reflected negatively on his/her academic achievement?

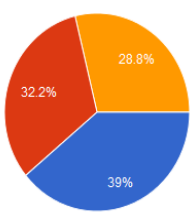

- Yes

No

Sometimes

Figure 5: Questionnaire asked parent “Would you use our suggested system?”

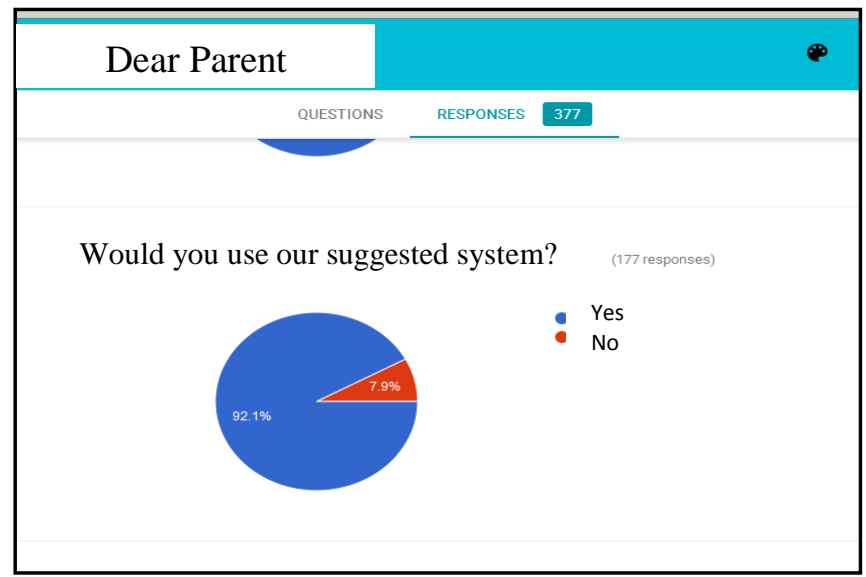

\section{CONCLUSION}

In this paper, a homework system is suggested in order to support Saudi Arabia's Vision 2030 in transmitting from using traditional learning methods to contemporary learning methods. In sequence, to check the impact of our suggested homework system, a questionnaire was developed. In recent years, involving parents in children's education is being placed in the educational process; therefore, Respondents of our developed questionnaire included teachers and parents. Although both teachers and parents have been asked by our developed questionnaire, the questionnaire has been more attractive for parents $(58.2 \%)$ comparing with teachers $(41.8 \%)$. The end-users of the suggested homework system will be parents, teachers, and administrators. In summation, Polls show that both parents and teachers are willing to use our suggested homework system; moreover, teachers are enthusiastic to discontinue using the traditional methods in teaching. Our suggested homework system will support vision 2030 to be well applied on technological basis. 


\section{REFERENCES}

[1] Greenfeld, Karl Taro, “My Daughter's Homework Is Killing Me,” The Atlantic, September 18, 2013

[2] Neil McNerney, “Homework: A Parent's Guide To Helping Out Without Freaking Out!," integrated publishing 1984 Isaac, Newton square, suite 204, December 26, 2011

[3] Cathy Vatterott, "Rethinking HOMEWORK Best Practices That Support Diverse Needs," ; ASCD, Alexandria, Virginia USA,2009

[4] https://sacm.org.au/saudi-vision-2030-envisages-a-thriving-education-research-and-renewable-energy-sectors/?refreshed.

[5] Cathy Vatterott, "Making Homework Central to Learning," ASCD, November 2011

[6] http://www.tamimi.com/en/magazine/law-update/section-14/august-2/vision-2030-and-the-transformation-of-education-in-saudiarabia.html

[7] Wendy Olsen, "Data Collection -Key Debates and Methods in social Resaerch," SAGE publications.Inc, 2012 